\title{
A different recruitment of the lateral and basolateral amygdala promotes contextual or elemental conditioned association in Pavlovian fear conditioning
}

\author{
Ludovic Calandreau, ${ }^{1}$ Aline Desmedt, ${ }^{1,2}$ Laurence Decorte, and Robert Jaffard \\ Laboratoire de Neurosciences Cognitives, Centre National de la Recherche Scientifique UMR 5106, Université de Bordeaux I, \\ 33405 Talence, France
}

\begin{abstract}
Convergent data suggest dissociated roles for the lateral (LA) and basolateral (BLA) amygdaloid nuclei in fear conditioning, depending on whether a discrete conditioned stimulus (CS)-unconditional stimulus (US) or context-US association is considered. Here, we show that pretraining inactivation of the BLA selectively impaired conditioning to context. In contrast, inactivation of the LA disrupted conditioning to the discrete tone CS, but also either impaired or enhanced contextual conditioning, depending on whether the context was in the foreground or in the background. Hence, these findings refine the current model of the amygdala function in emotional learning by showing that the BLA and the LA not only differentially contribute to elemental and context-US association, but also promote, through their interaction, the most relevant of these two associations.
\end{abstract}

Consensus has emerged concerning the involvement of the amygdala in mediating appropriate emotional behavior (Bechara et al. 1995; LaBar et al. 1995; LeDoux and Muller 1997; LeDoux 2000). For instance, lesions to the amygdala have been shown to disrupt conditioned fear responses (LeDoux et al. 1990; Maren et al. 1996; Cousens and Otto 1998; Maren 1999) that typically constitute a defense reaction elicited by a discrete conditioned stimulus (CS, e.g., a tone) previously paired with an aversive unconditional stimulus (US, e.g., foot-shock). It is also well established that the amygdala has an indispensable role in contextual fear conditioning, a situation in which conditioned fear responses are elicited by various static contextual stimuli present in the training environment. However, the amygdala is anatomically heterogeneous and the contribution of different amygdaloid nuclei to these different types of fear conditioning remains elusive. In a current theoretical model developed by LeDoux (2000), the basolateral amygdala complex (BLC), defined as the set of lateral (LA), basolateral (BLA), and basomedial (BM) nuclei, is known to play a critical role in both elemental (discrete auditory-cue CS-US association) and contextual fear conditioning. In particular, several lines of investigation have highlighted the crucial role of the LA in elemental fear conditioning. Two circuits, including either the direct projections from the thalamus or the projections from the auditory cortex to the LA are thought to be critical for auditory-cue CS-US associations (Romanski and LeDoux 1992; Li et al. 1996; LeDoux and Muller 1997). Moreover, lesions of the LA, but not of the BLA, accessory basal, or medial nucleus of the amygdala block auditory-cue conditioning (Nader et al. 2001).

In contrast, amygdaloid involvement in contextual conditioning is poorly understood. According to a current view, the hippocampus would be required for forming an integrated representation of the context (Fanselow 2000), which then comes

1These two authors contributed equally to this work.
2Corresponding author.

E-mail a.desmedt@Inc.u-bordeaux1.fr; fax +33 540008743.

Article published online ahead of print. Article and publication date are at http://www.learnmem.org/cgi/doi/10.1101/lm.92305. into association with the US in the amygdala (Maren 2001). This implies that projections from the hippocampal formation (HF) are required for processing context-US associations (Selden et al. 1991; Phillips and LeDoux 1992). The BLC, and the BLA nucleus in particular, constitutes one of the major efferent loci for these projections (Canteras and Swanson 1992; Maren 1996). In this respect, it has been shown that HF-BLC (among which, presumably, HF-BLA) synapses sustained long-term potentiation (LTP) (Maren and Fanselow 1995) in a similar manner as thalamo-LA synapses (Rogan et al. 1997). However, the respective roles of the LA and the BLA in contextual conditioning have not yet been specified.

In order to address this issue, we have compared, in mice, the effects of reversible neural inactivation of either the LA or the BLA on two types of fear conditioning known to result in different contextual processing (i.e., background versus foreground contextual conditioning) (Phillips and LeDoux 1994). Since these two conditioning procedures (i.e., CS-US paired versus CS-US unpaired) are supposed to result in either a predominant discrete CS-US or context-US association (Desmedt et al. 1998, 1999), such an experimental design further enables us to directly assess the relative contribution of these two amygdaloid nuclei to elemental and contextual conditioning.

\section{Results}

\section{Histology}

An illustration of injection sites and approximate diffusional area of the injectate, which was found to overlap the targeted nucleus (BLA or LA), are shown in Figure 1. Only data from subjects that exhibited correctly placed injection sites were used in the analysis. Among a total of 110 animals, 14 mice exhibited either dislocated cannulae placements relative to the targeted nucleus or injection sites that reached the central nucleus. All of those subjects that exhibited injection sites involving possible drug diffusion to a nucleus other than the one of interest were excluded from the analysis. 


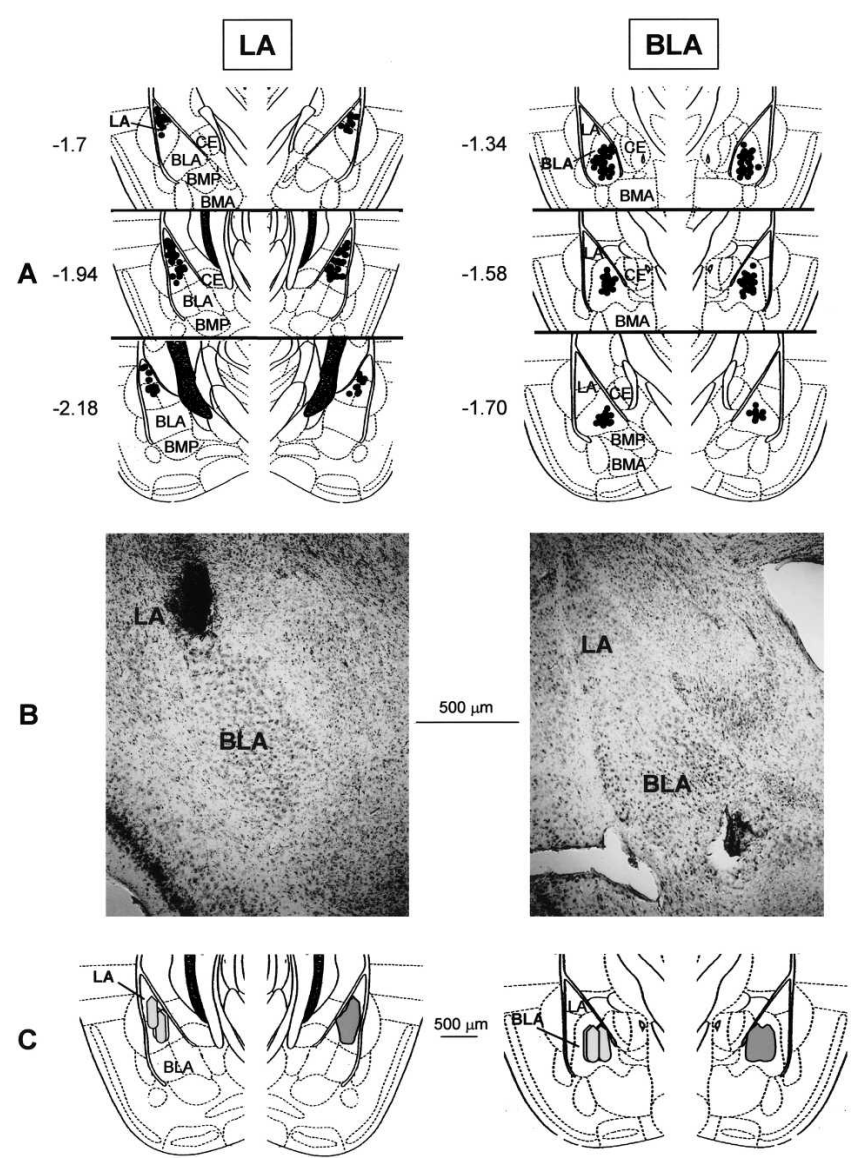

Figure 1. Histological controls. (A) Illustration of the injectors' tip location within the LA (left) and the BLA (right). Numbers indicate relative position of the coronal sections in millimeters posterior to bregma (adapted with permission from Elsevier (C) 1997, Franklin and Paxinos 1997). (BLA) Basolateral nucleus; (BMA) basomedial nucleus, anterior; (BMP) basomedial nucleus, posterior; (LA) lateral nucleus; (CE) central nucleus. (B) Representative photomicrograph depicting the cannulae placement into the LA (left) and the BLA (right). (C) Schematic drawing of coronal sections of a mouse brain hemisphere $(-1.94$ [left] and -1.58 [right] posterior to bregma), showing the sphere of diffusion of a $0.15-\mu \mathrm{L}$ solution of India ink into the LA (left) or the BLA (right) under the conditions as detailed in Materials and Methods. For each coronal section, the gray area (right part of the section) represents the superimposed sphere of diffusion in a sample of four animals that were either LA- (left) or BLA(right) infused mice.

\section{Behavioral results}

Mice were bilaterally infused with either lidocaine or $\mathrm{NaCl}$ (or pseudo-infused) into the BLA or into the LA just prior to training for fear conditioning. During training, they received either paired tone CS-US presentations (i.e., with the conditioning context in the background) or unpaired tone CS-US presentations (i.e., context in the foreground). Twenty-four hours later, all animals were tested for conditioned freezing to the tone CS (auditory-cue test), and $2 \mathrm{~h}$ later for conditioned freezing to the conditioning context (context test) (Fig. 2).

\section{Auditory-cue conditioning}

Overall conditioned freezing to the tone CS (Fig. 3) was specific both to the paired (versus unpaired) conditioning procedure and to tone presentation ( $2^{\text {nd }}$ versus $1^{\text {st }}$ block) [block $\times$ conditioning interaction: $F(1,84)=39.85, P<0.001]$. As compared with their matched controls (pseudo- and NaCl-infused groups), mice in- fused with lidocaine into the LA prior to training with the paired procedure were impaired in conditioned freezing to the tone CS (Fig. 3B), whereas those infused into the BLA were not (Fig. 3A) [block $\times$ treatment $\times$ nucleus triple interaction: $F\left({ }_{2,45}\right)=3.99$, $P=0.025]$. Namely, the specific increase in freezing observed between the $1^{\text {st }}$ and $2^{\text {nd }}$ blocks in control mice trained with the paired (versus unpaired) procedure [block $\times$ conditioning interaction: $F(1,56)=28.73, P<0.001]$ was totally abolished by inactivation of the LA [interaction: $F\left({ }_{1,14}\right)=1.03, \mathrm{~ns}$ ], but fully preserved when inactivation was targeted to the BLA [interaction: $F(1,14)=12.08, P=0.003]$. Otherwise, inactivating either the LA or the BLA prior to training with the unpaired procedure did not affect the subsequent amount of freezing recorded during the auditory-cue test (all $F$ values $<1$ ). These results demonstrate that the LA, but not the BLA, is necessary for the acquisition of auditory-cue conditioning.

\section{Contextual conditioning}

Control mice (pseudo- and $\mathrm{NaCl}$-infused mice) displayed significantly more conditioned freezing to the context when trained with the unpaired (context in the foreground) than with the paired procedure (context in the background) [effect of conditioning: $\left.F\left({ }_{1,56}\right)=42.61, P<0.0001\right]$. Changes in contextual freezing produced by lidocaine infusions were both dependent on the targeted nucleus (LA versus BLA) and on the conditioning procedure used [conditioning $\times$ treatment $\times$ nucleus triple interaction: $F(2,84)=4.59, P=0.012$; see Fig. 4].

\section{General impairment after BLA inactivation}

Mice infused with lidocaine into the BLA (Fig. 4A) were impaired in contextual freezing [effect of treatment: $F\left({ }_{2,41}\right)=14.78$, $P<0.001]$ irrespective of the conditioning procedure used [conditioning $\times$ treatment interaction: $F(2,41)=1.79$, ns]. These results demonstrate that the BLA is necessary for both background and foreground contextual conditioning.

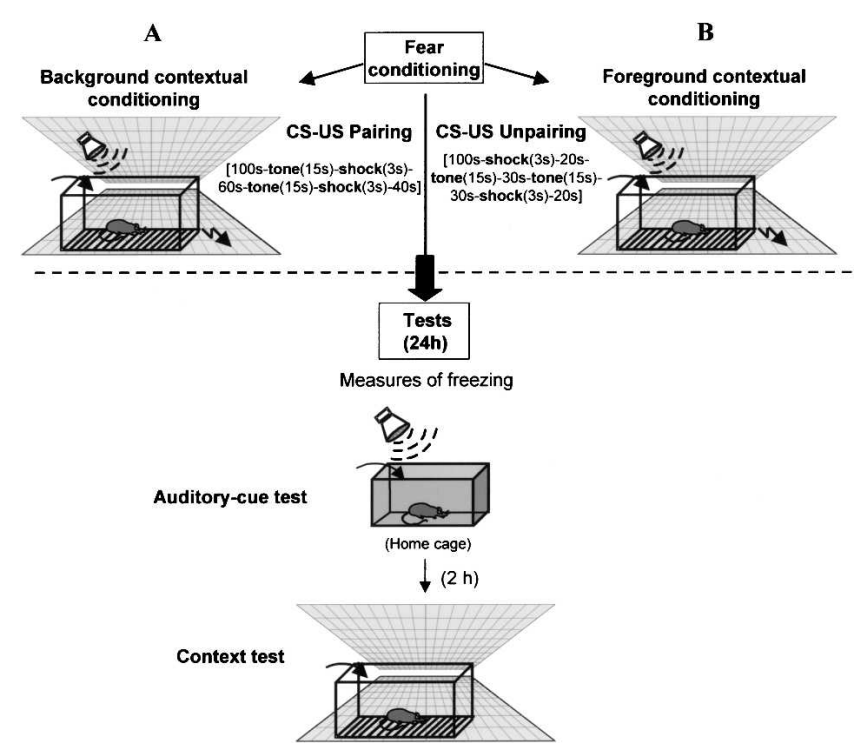

Figure 2. Behavioral procedure. For acquisition of fear conditioning, animals were either submitted to an auditory-cue conditioning, which consists in tone CS-footshock-US pairing (background contextual conditioning) (A), or to a CS-US unpairing (foreground contextual conditioning) $(B)$. Then, animals were replaced in their home cages. Twenty-four hours later, each animal was first submitted to the auditory-cue test while maintained in its home cage, then ( $2 \mathrm{~h}$ later) was re-exposed to the conditioning context for the context test. During each of these tests, the amounts of freezing were assessed by 2-min blocks. 


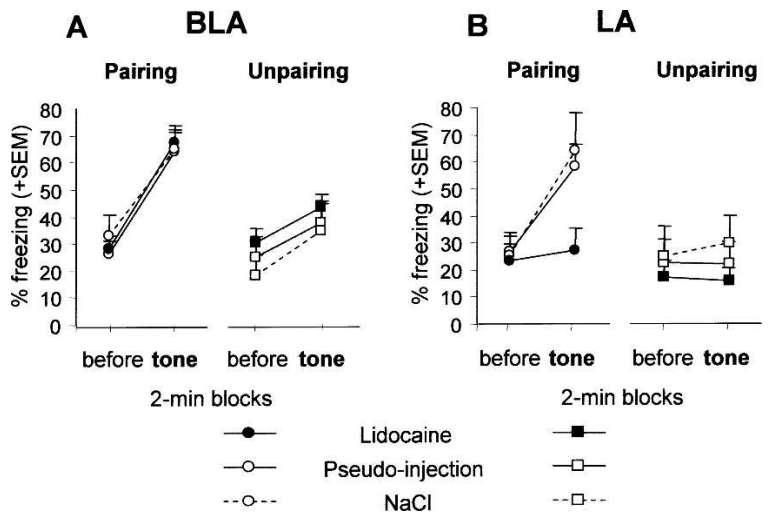

Figure 3. Auditory-cue test. Mean percentage freezing ( \pm SEM) on successive 2-min blocks before and during tone presentation in animals that were submitted to lidocaine infusion $(n=32), \mathrm{NaCl}(n=31)$, or pseudo-infusion $(n=33)$ into the BLA $(A)$ or into the LA $(B)$, before either CS-US pairing or CS-US unpairing.

\section{Contrasted effects of LA inactivation}

In mice infused with lidocaine into the LA (Fig. 4B), contextual freezing was either impaired or enhanced, depending on whether animals were trained with the unpaired or with the paired procedure [conditioning $\times$ treatment interaction $F(2,43)=17.37$, $P<0.0001]$. Namely, mice infused with lidocaine into the LA and trained with the unpaired procedure displayed a significant impairment of contextual freezing with respect to each control group [pseudo infusions: $F\left({ }_{1,14}\right)=41.63, P<0.0001$; $\mathrm{NaCl}$ infusions: $\left.F\left({ }_{1,13}\right)=5.46, P=0.036\right]$. In contrast, the same lidocaine infusion, but in animals trained with the paired procedure resulted in a significant enhancement of freezing with respect to controls $\left[F\left({ }_{1,17}\right)=9.45, P<0.01\right.$ and $F\left({ }_{1,13}\right)=5.99, P=0.029$, respectively]. These results demonstrate that the LA is involved in contextual conditioning, but that the direction of this involvement (i.e., facilitation versus inhibition of conditioned freezing) may change according to the conditioning procedure used.

Hence, in foreground contextual conditioning (unpaired procedure), lidocaine-infused mice displayed an impaired freezing to contextual cues whatever the targeted nucleus [nucleus $\times$ treatment interaction: $F(2,39)=1.75, \mathrm{~ns}$, whereas in background contextual conditioning (paired procedure), freezing to the context was either impaired or enhanced, depending on whether the inactivated nucleus was the BLA or the LA, respectively [nucleus $\times$ treatment interaction: $F(2,45)=9.12, P=0.005]$.

\section{Discussion}

Numerous experiments have shown that the BLC is critically involved in the acquisition of both cued (elemental) and contextual fear conditioning (Fanselow and Kim 1994; Helmstetter and Belgowan 1994; Muller et al. 1997; Fanselow and LeDoux 1999; Wilensky et al. 1999; Goosens and Maren 2001). However, these studies have not provided any direct evidence for a distinct role of the BLA and the LA nuclei in these two components of conditioned fear behavior. This is probably because most of the amygdalar damage/ inactivation extended to the whole BLC, and/or because analysis was restricted to

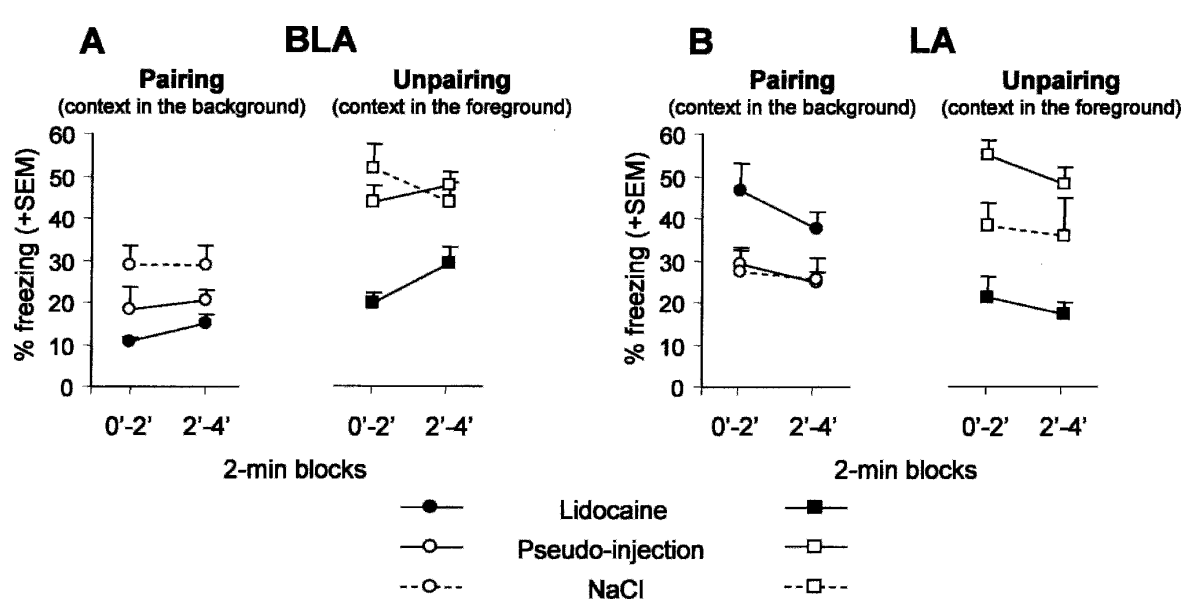

Figure 4. Context test. Mean percentage freezing ( \pm SEM) on successive 2-min blocks during reexposure to the conditioning context in animals that were submitted to lidocaine, $\mathrm{NaCl}$, or pseudoinfusion into the BLA $(A)$ or into the LA $(B)$, before either CS-US pairing or CS-US unpairing. either cued or contextual conditioning. However, one study showed that lesions restricted to the LA, but not those restricted to the BLA, impaired auditory-cue fear conditioning (Nader et al. 2001). While confirming this functional dissociation, the present study showed that, irrespective of the conditioning procedure used (i.e., tone CS-US paired or unpaired), inactivation of the BLA selectively impaired contextual fear conditioning. Moreover, inactivating the LA was found not only to disrupt auditory-cue conditioning, but also to either impair or enhance contextual conditioning, depending on whether the context was in the foreground (unpaired procedure) or in the background (paired procedure). Thus, in contrast to the BLA, the role of which would be restricted to contextual conditioning, the LA (1) is involved in both types of conditioning (auditory-cued and contextual), and (2) its contribution to contextual conditioning would change depending on the relative predictability of static contextual cues (with respect to the phasic-tone CS) for signaling the occurrence of the US.

From a pharmacological point of view, several observations indicate that the active site of the injected volume of lidocaine was limited either to the LA or the BLA. First, previous technical (Peterson 1998) and functional (Malpeli and Schiller 1979) studies examining the diffusion of microinjected drugs into discrete brain areas, allowed us to estimate that a $0.15-\mu \mathrm{L}$ volume administered over $3 \mathrm{~min}$ resulted in a sphere of functional inactivation of $\sim 200-400 \mu^{3}$ in the brain. In our experiment, this implies that the drug spread was mostly limited to the targeted region. Second and more importantly, the observed dissociation of the behavioral effects of the injections depending on the targeted nucleus indicates that there exists only minimal overlap, if indeed there was any overlap at all between the two spheres of drug diffusion.

\section{Role of the BLA}

While being totally inefficient in altering auditory-cue fear conditioning, inactivation of the BLA selectively impaired fear conditioning to context. This impairing effect was more pronounced when the context was in the foreground than in the background. However, this difference might well be accounted for by a floor effect (Fig. 4A), due to the overshadowing of contextual cues by the predictive-tone CS (see Marlin 1981). The selective involvement of the BLA in contextual conditioning is congruent with findings from anatomical and electrophysiological studies. Namely, a prominent source of BLA afferents is the hippocampal formation with projections arising from the hippocampal 
CA1, the ventral subiculum, and the lateral entorhinal cortex, and coursing through the ventral angular bundle (Ottersen 1982; Canteras and Swanson 1992). Such projections are thought to be required for contextual, but not elemental conditioning (e.g., Phillips and LeDoux 1992). In contrast, the pathways through which auditory CS inputs arising from the medial geniculate and temporal neocortical areas reach the amygdala points to the LA as the main, if not exclusive, target (Romanski et al. 1993; Maren 1996; LeDoux and Muller 1997). In addition, it has been shown that electrical stimulation of the ventral perirhinal cortex was able to induce long-term potentiation in the BLA, but not in the LA, whereas electrical stimulation of the medial geniculate nucleus produced just the opposite pattern of effect (Yaniv et al. 2001). Altogether, these findings suggest that, within the BLC, there would be a segregation between synapses involved in the processing of context-US association (i.e., within the BLA) and those involved in the processing of tone CS-US association (i.e., within the LA). Hence, the dissociation presently observed is in agreement with the theoretical model proposed by LeDoux $(1993,2000)$, which suggests dissociated roles for the LA and the BLA in elemental and contextual fear conditioning, respectively. However, the pattern of effects produced by LA inactivation on both elemental and contextual conditioning points toward a more complex functional scheme.

\section{Role of the LA in contextual conditioning}

Although LA-inactivated mice did not exhibit conditioned freezing to the tone CS, these mice still displayed different amounts of conditioned freezing to the context as a function of the training (i.e., CS-US paired versus unpaired) procedure. However, in contrast to controls that exhibited higher levels of foreground than background contextual freezing, LA-inactivated mice displayed an opposite pattern of responding (Fig. 4B). Two important conclusions follow from these observations. First, the LA cannot be the only brain site where tone CS-US contingencies are processed. Indeed, LA-inactivated mice did discriminate between the two training conditions as assessed by their levels of contextual freezing. This finding is in line with previous data showing that despite amygdalar damage, animals still displayed neural activities or conditioned responses dependent on the CS-US contingency (Killcross et al. 1997; Desmedt et al. 1998). Second, the LA is nevertheless necessary for adapting the magnitude of contextual freezing at the appropriate level, that is, in relation to the contingency between the tone CS and the US. Finally, it must be re-emphasized that, in contrast to the BLA, the inactivation of which impaired contextual conditioning in both training conditions, inactivation of the LA either enhanced or impaired such conditioning, depending on whether the context was in the background or in the foreground, respectively. This indicates that, depending on whether the occurrence of the US is (or is not) predicted in a precise (explicit) time-dependent manner by the tone CS, the processing of contextual information is subserved by a differential recruitment of the LA and the BLA.

\section{LA and background contextual conditioning}

With the context in the background, inactivation of the LA prior to training resulted in an enhancement of contextual conditioned freezing. The question then arises as to how such facilitation may occur. Multiple-systems theories of brain function generally consider that facilitation of performance in a learning task following brain damage occurs because of the weakening (or suppression) of a process (or a "solution"), which competes with another one to solve the problem at hand (Jaffard and Meunier 1993; Kim and Baxter 2001). Thus, increased conditioning to background contextual cues would thus occur, because preventing the phasic-tone CS to enter into association with the US within the LA would unmask (facilitate) the BLA-mediated context-US association. Hence, we suggest that in this learning situation (i.e., CS-US paired), the LA would not only process the tone CS-US association, but would also inhibit (or compete with) the processing of context-US association by the BLA.

\section{LA and foreground contextual conditioning}

With the context in the foreground, inactivation of the LA produced the same degree of impairment of contextual conditioning as the inactivation of the BLA. This indicates that both nuclei are required for an optimal level of fear conditioning to context, suggesting thereby a synergistic contribution of LA- and BLAmediated processes to contextual conditioning. The observation that LA inactivation impairs both elemental and foreground contextual conditioning could indicate that the LA contributes to the association based on the more predictive information for the occurrence of the US (i.e., the tone CS in the CS-US paired condition and the context in the unpaired condition, respectively). More parsimoniously, the LA may contribute to contextual conditioning by promoting elemental associations between individual static contextual cues and the US. This is congruent with data suggesting that the context could be processed as individual elements or as a unified representation of environmental cues (Frankland et al. 1998; Gerlai 1998; Cho et al. 1999; Anagnostaras et al. 2001). Thus, the impairing effect of LA inactivation on fear conditioning to both the foreground context and the explicit-tone CS could result from the same basic impairment in forming elemental CS (either phasic or static)-US associations.

Whatever be the case, the contrasted pattern of effects of LA and BLA inactivation on contextual conditioning (i.e., similar impairing effect on foreground versus opposite effect on background contextual conditioning) suggests that decreasing the predictive value of the discrete-tone CS (i.e., from CS-US pairing to CS-US unpairing) changes the way the BLA- and LA-mediated processes interact, that is, from competition (high-predictive value) to synergy (low-predictive value).

In conclusion, selective neural inactivation of either the BLA or the LA has revealed dissociated roles for these amygdaloid nuclei in contextual and elemental fear conditioning. These findings are congruent with anatomical and electrophysiological data suggesting that heaviest projections to LA are thought to convey signals subserving simple/unimodal stimuli and would be gradually replaced by more complex polymodal projections when moving toward BLA (Gloor 1997; Yaniv et al. 2001, 2004). However, the present study further demonstrates that the LA is also involved in contextual conditioning, and that this involvement is dependent on the training procedure (i.e., foreground versus background contextual conditioning). This led us to refine the standard model (LeDoux 1993, 2000) describing LA/BLA recruitment as subserving Pavlovian fear conditioning. We propose that, depending on the predictive value of the discrete-tone CS for the occurrence of the US, LA-mediated processes would either compete (tone CS-US paired) or cooperate (tone CS-US unpaired) with BLA-mediated processes, thereby promoting, in both cases, the most relevant association (tone CS-US or context-US). Although further experiments are needed to unveil the mechanisms subserving such a differential interaction, the present findings highlight the BLA and LA contributions to appropriate emotional responses as a function of the particular learning situation considered. 


\section{Materials and Methods}

\section{Subjects and surgery}

The experiments were conducted on naive adult (4-6 mo) male mice (C57Bl/6 JI Co, IFFA Credo) weighing 27-32 g. They were housed individually $7 \mathrm{~d}$ before the experiment and maintained in an animal room $\left(23^{\circ} \mathrm{C}\right)$ with a 12 -h artificial light/dark cycle and with ad libitum access to food and water.

Mice were anesthetized with avertin (tribromoethanol; 10 $\mathrm{mL} / \mathrm{kg}$, i.p.) and secured in a Narishige stereotaxic apparatus. Stainless-steel guide cannulae (26 gauge, 8-mm length) were implanted bilaterally either $1 \mathrm{~mm}$ above the BLA (3900 $\mu \mathrm{m}$ below the skull surface, $1400 \mu \mathrm{m}$ posterior to bregma, and $2900 \mu \mathrm{m}$ lateral from midline), or $1 \mathrm{~mm}$ above the LA (3250 $\mu \mathrm{m}$ below the skull surface, $2000 \mu \mathrm{m}$ posterior to bregma, and $3250 \mu \mathrm{m}$ lateral from midline) (Franklin and Paxinos 1997), then fixed in place with dental cement and two jewel screws attached to the skull. Mice were then allowed to recover in their home cages in the animal room for at least $8 \mathrm{~d}$ before behavioral experiment.

\section{Intra-cerebral injections}

For injection, the dummy stylets that obturated the free end of the guide cannulae were removed, and 32-gauge stainless-steel cannulae $(9 \mathrm{~mm})$ attached to $1-\mu \mathrm{L}$ Hamilton syringes (PolyLabo) with polyethylene catheter tubing were inserted through the guides. The syringes were fixed in a constant rate infusion pump (Roucaire), and 2\% lidocaine (2-diethylamino-N-[2,6-dimethylphenyl]-acetamide hydrochloride, Sigma) was bilaterally infused $(0.15 \mu \mathrm{L}$ per side) either into the BLA or the LA over a 3-min period, during which the mouse was maintained in its home cage. Infusion flow rates were checked visually. The cannulae were left in place for a further $3 \mathrm{~min}$ before removal to allow diffusion of the drug away from the cannulae tip (Desmedt et al. 1999).

The injection volume was chosen on the basis of previous findings reporting that this resulted in selective functional inactivation of $200-400 \mu^{3}$ brain regions (Malpeli and Schiller 1979), that is, in our experiment, a drug spread limited to the targeted region. By inducing a peak of inactivation within the targeted brain site lasting from 3 to $10 \mathrm{~min}$, this pre-training infusion was supposed to induce a functional inactivation of either the BLA or the LA that was limited mostly to the acquisition phase of conditioning. Two control groups were submitted to the same procedure, except that animals either received $\mathrm{NaCl}$ infusion, or no infusion (pseudo-infusion). The sphere of diffusion of the injection volume was estimated in a set of animals microinjected with $0.15 \mu \mathrm{L}$ of India ink either into the LA or the BLA.

\section{Behavioral procedure}

Once transferred to the experimental room, mice were submitted either to lidocaine, $\mathrm{NaCl}$ infusions, or pseudo-infusions $5 \mathrm{~min}$ before acquisition of conditioning. Classical fear conditioning took place in a Plexiglas box $(30 \times 24 \times 22 \mathrm{~cm}$ high $)$ affording access to the visuo-spatial cues in the experimental room. The floor of this conditioning chamber consisted of stainless steel rods ( $2 \mathrm{~mm}$ diameter), spaced $5 \mathrm{~mm}$ apart and connected to a shock generator (Imetronic). The four sides of the chamber and the rods of the floor were cleaned with $90 \%$ ethanol before each trial. For acquisition, half of the animals were submitted to two tone CS $(63 \mathrm{~dB}, 1 \mathrm{kHz}, 15 \mathrm{sec})$-footshock US $(0.9 \mathrm{~mA}, 50 \mathrm{~Hz}, 3 \mathrm{sec})$ pairings (Fig. 2A). In this training condition, the prevalent association is the elemental CS-US association, which consigns the conditioning context to the background. The other half was submitted to tone-shock unpairing; the only difference with the previous training condition was that the two shocks and the two tones were pseudo-randomly distributed (Fig. 2B). This explicit unpairing of CS and US is known to make the contextual cues the primary stimuli that enter into association with the US (i.e., context in the foreground) (Phillips and LeDoux 1994; Desmedt et al. 1999).
Twenty-four hours later, while mice were drug free, they were tested for freezing behavior (measured second-by-second), used as an index of conditioned fear, and defined as a lack of all movement except for respiratory-related movements (Blanchard and Blanchard 1969). First, mice were submitted to the auditorycue test; they were maintained in their home cage, and two successive recording sessions of behavioral responses were performed prior to (first $2 \mathrm{~min}$ ) and during (next $2 \mathrm{~min}$ ) tone presentation. Two hours later, mice were submitted to the context test; they were re-exposed to the conditioning chamber and two successive 2-min recordings of behavioral responses were performed. Animals were continuously videotaped for off-line scoring of freezing.

\section{Histology}

After completion of the behavioral study, animals were given an overdose of sodium thiopental $(120 \mathrm{mg} / \mathrm{kg})$ and transcardially perfused with physiological saline, followed by $10 \%$ buffered formalin. Brains were post-fixed in formalin-saccharose 30\% solution for $1 \mathrm{wk}$, frozen, cut coronally on a sliding microtome into $60-\mu \mathrm{m}$ sections that were mounted on a gelatin-coated slide, and stained with thionine in order to evaluate the cannulae placements.

\section{Data analysis}

Freezing was calculated as the percentage $( \pm$ SEM $)$ of the total time spent freezing during every 2-min recording session. Statistical analysis of the data was performed using analysis of variance (ANOVA) (Systat).

\section{Acknowledgments}

We thank Dr. A. Marighetto and Dr. T.P. Durkin for linguistic corrections of the manuscript and helpful discussions. This study was supported by the Centre National de la Recherche Scientifique.

\section{References}

Anagnostaras, S.G., Gale, G.D., and Fanselow, M.S. 2001. Hippocampus and contextual fear conditioning: Recent controversies and advances. Hippocampus 11: 8-17.

Bechara, A., Tranel, D., Damasio, H., Adolphs, R., Rockland, C., and Damasio, A.R. 1995. Double dissociation of conditioning and declarative knowledge relative to the amygdala and hippocampus in humans. Science 269: 1115-1118.

Blanchard, R.J. and Blanchard, D.C. 1969. Crouching as an index of fear. J. Comp. Physiol. Psychol. 67: 370-375.

Canteras, N.S. and Swanson, L.W. 1992. Projections of the ventral subiculum to the amygdala, septum, and hypothalamus: A PHAL anterograde tract-tracing study in the rat. J. Comp. Neurol. 324: $180-194$.

Cho, Y.H., Friedman, E., and Silva, A.J. 1999. Ibotenate lesions of the hippocampus impair spatial learning but not contextual fear conditioning in mice. Behav. Brain. Res. 98: 77-87.

Cousens, G. and Otto, T. 1998. Both pre- and post-training excitotoxic lesions of the basolateral amygdala abolish the expression of olfactory and contextual fear conditioning. Behav. Neurosci. 112: 1092-1103.

Desmedt, A., Garcia, R., and Jaffard, R. 1998. Differential modulation of changes in hippocampal-septal synaptic excitability by the amygdala as a function of either elemental or contextual fear conditioning in mice. J. Neurosci. 18: 480-487.

. 1999. Vasopressin in the lateral septum promotes elemental conditioning to the detriment of contextual fear conditioning in mice. Eur. J. Neurosci. 11: 3913-3921.

Fanselow, M.S. 2000. Contextual fear, gestalt memories, and the hippocampus. Behav. Brain Res. 110: 473-486.

Fanselow, M.S. and Kim, J.J. 1994. Acquisition of contextual Pavlovian fear conditioning is blocked by application of an NMDA receptor antagonist D,L-2-amino-5-phosphonovaleric acid to the basolateral amygdala. Behav. Neurosci. 108: 210-212.

Fanselow, M.S. and LeDoux, J.E. 1999. Why we think Plasticity underlying Pavlovian fear conditioning occurs in the basolateral amygdala. Neuron 23: 229-232.

Frankland, P.W., Cestari, V., Filipkovski, R.K., McDonald, R.J., and Silva, A.J. 1998. The dorsal hippocampus is essential for context 
discrimination but not for contextual conditioning. Behav. Neurosci. 112: $863-874$.

Franklin, K.B.J. and Paxinos, G. 1997. The mouse brain in stereotaxic coordinates. Academic Press, New York.

Gerlai, R. 1998. Contextual learning and cue association in fear conditioning in mice: A strain comparison and a lesion study. Behav. Brain. Res. 95: 191-203.

Gloor, P. 1997. The temporal lobe and limbic system. Oxford University Press, New York.

Goosens, K.A. and Maren, S. 2001. Contextual and auditory fear conditioning are mediated by the lateral, basal, and central amygdaloid nuclei in rats. Learn. Mem. 8: 148-155.

Helmstetter, F.J. and Bellgowan, P.S. 1994. Effects of muscimol applied to the basolateral amygdala on acquisition and expression of contextual fear conditioning in rats. Behav. Neurosci. 108: $1005-1009$.

Jaffard, R. and Meunier, M. 1993. Role of the hippocampal formation in learning and memory Hippocampus 3: 203-217.

Killcross, S., Robbins, T.W., and Everitt, B.J. 1997. Different types of fear-conditioned behaviour mediated by separate nuclei within amygdala. Nature 388: $377-380$.

Kim, J.J. and Baxter, M.G. 2001. Multiple brain-memory systems: The whole does not equal the sum of its parts. Trends Neurosci. 24: 324-330.

LaBar, K.S., LeDoux, J.E., Spencer, D.D., and Phelps, E.A. 1995. Impaired fear conditioning following unilateral temporal lobectomy in humans. J. Neurosci. 15: 6846-6855.

LeDoux, J.E. 1993. Emotional memory systems in the brain. Behav. Brain. Res. 58: $69-79$.

.2000. Emotion circuits in the brain. Annu. Rev. Neurosci. 23: $155-184$

LeDoux, J.E. and Muller, J. 1997. Emotional memory and psychopathology. Phil. Trans. R. Soc. Lond. 352: 1719-1726.

LeDoux, J.E., Cicchetti, P., Xagoraris, A., and Romanski, L.M. 1990. The lateral amygdaloid nucleus: Sensory interface of the amygdala in fear conditioning. I. Neurosci. 10: 1062-1069.

Li, X., Stutzman, G.E., and LeDoux, J.E. 1996. Convergent but temporally separated inputs to the lateral amygdala neurons from the auditory thalamus and auditory cortex use different postsynaptic receptors: In vivo intracellular and extracellular recordings. Learn. Mem. 3: 229-242.

Malpeli, J.G. and Schiller, P.H. 1979. A method of reversible inactivation of small regions of brain tissue. J. Neurosci. Meth. 1: 143-151.

Maren, S. 1996. Synaptic transmission and plasticity in the amygdala, an emerging physiology of fear conditioning circuits. Mol. Neurobiol. 13: $1-22$.

- 1999. Neurotoxic basolateral amygdala lesions impair learning and memory but not the performance of conditional fear in rats. $J$. Neurosci. 19: 8696-8703.

. 2001. Neurobiology of Pavlovian fear conditioning. Аnnu. Rev. Neurosci. 24: 897-931.

Maren, S. and Fanselow, M.S. 1995. Synaptic plasticity in the basolateral amygdala induced by hippocampal formation stimulation in vivo. $J$. Neurosci. 15: $7548-7564$.
Maren, S., Aharonov, G., and Fanselow, M.S. 1996. Retrograde abolition of conditional fear after excitotoxic lesions in the basolateral amygdala of rats: Absence of temporal gradient. Behav. Neurosci. 110: $718-726$

Marlin, N.A. 1981. Contextual associations in trace conditioning. Anim. Learn. Behav. 9: 519-523.

Muller, J., Corodimas, K.P., Fridel, Z., and LeDoux, J.E. 1997. Functional inactivation of the lateral and basal nuclei of the amygdala by muscimol infusion prevents fear conditioning to an explicit conditioned stimulus and to contextual stimuli. Behav. Neurosci. 111: $683-691$.

Nader, K., Majidishad, P., Amorapanth, P., and LeDoux J.E. 2001. Damage to the lateral and central, but not other, amygdaloid nuclei prevents the acquisition of auditory fear conditioning. Learn. Mem. 8: $156-163$

Ottersen, O.P. 1982. Connections of the amygdala of the rat. IV: Corticoamygdaloid and intraamygdaloid connections as studied with axonal transport of horseradish peroxidase. J. Comp. Neurol. 205: $30-48$.

Peterson, S.L. 1998. Drug microinjection in discrete brain regions. Kopf Carrier 50: 1-6.

Phillips, R.G. and LeDoux, J.E. 1992. Differential contribution of amygdala and hippocampus to cued and contextual fear conditioning. Behav. Neurosci. 106: 274-285.

. 1994. Lesions of the dorsal hippocampal formation interfere with background but not foreground contextual fear conditioning. Learn. Mem. 1: 34-44.

Rogan, M.T., Stäubli, U.V., and LeDoux, J.E. 1997. Fear conditioning induces associative long-term potentiation in the amygdala. Nature 390: 604-607.

Romanski, L.M. and LeDoux, J.E. 1992. Equipotentiality of thalamo-amygdala and thalamo-cortico-amygdala circuits in auditory fear conditioning. J. Neurosci. 12: 4501-4509.

Romanski, L.M., Clugnet, M.C., Bordi, F., and LeDoux, J.E. 1993. Somatosensory and auditory convergence in the lateral nucleus of the amygdala. Behav. Neurosci. 107: 444-450.

Selden, N.R.W., Everitt, B.J., Jarrard, L.E., and Robbins, T.W. 1991. Complementary roles for the amygdala and hippocampus in aversive conditioning to explicit and contextual cues. Neuroscience 42: $335-350$.

Wilensky, A.E., Schafe, G.E., and LeDoux, J.E. 1999. Functional inactivation of the amygdala before but not after auditory fear conditioning prevents memory formation. J. Neurosci. 19: RC48: $1-5$.

Yaniv, D., Schafe, G.E., LeDoux, J.E., and Richter-Levin, G. 2001. A gradient of plasticity in the amygdala revealed by cortical and subcortical stimulation, in vivo. Neuroscience 106: 613-620.

Yaniv, D., Desmedt, A., Jaffard, R., and Richter-Levin, G. 2004. The amygdala and appraisal processes: Stimulus and response complexity as an organizing factor. Brain Res. Brain Res. Rev. 44: 179-186.

Received February 1, 2005; accepted in revised form May 6, 2005. 


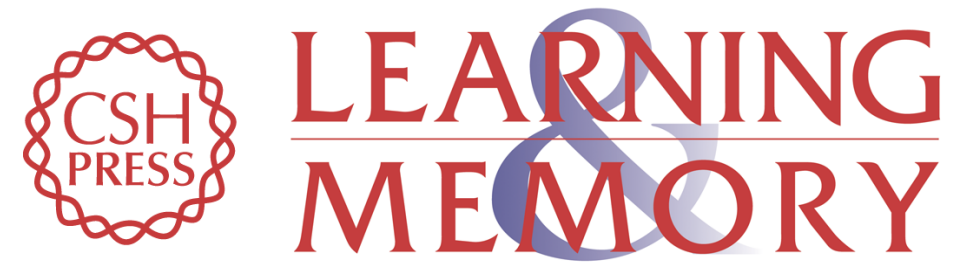

\section{A different recruitment of the lateral and basolateral amygdala promotes contextual or elemental conditioned association in Pavlovian fear conditioning}

Ludovic Calandreau, Aline Desmedt, Laurence Decorte, et al.

Learn. Mem. 2005, 12:

Access the most recent version at doi:10.1101//m.92305

References This article cites 41 articles, 11 of which can be accessed free at: http://learnmem.cshlp.org/content/12/4/383.full.html\#ref-list-1

License

Email Alerting

Receive free email alerts when new articles cite this article - sign up in the box at the Service top right corner of the article or click here. 